\title{
Diabetic autonomic neuropathies as a major risk factor for silent myocardial ischemia and infarction: a literature review
}

\author{
Kindo Shek Kamara $^{1 *}$, Tong ChuanFeng ${ }^{1}$ \\ ${ }^{1}$ Department of Cardiology, Zhongnan Hospital of Wuhan University, Wuhan, China \\ *Corresponding author E-mail:
}

\begin{abstract}
A substantial number of myocardial infarctions (MIs) are asymptomatic or associated with mi-nor and atypical symptoms. These symptoms are often atypical or even absent, especially in di-abetic patients. A number of studies on this topic have shown that in the presence of myocardial ischemia and infarction, diabetic patients report angina less frequently than non-diabetic pa-tients. In this article review, we researched several published articles written about this subject and investigated the relationship between Diabetes Mellitus (DM) in patients and the occur-rence of silent myocardial ischemia and infarction due to the onset of diabetic neuropathy (DN), which is brought about by prolonged diabetes. Based on our research of published articles and studies, we found overwhelming evidence that supports the fact that diabetic autonomic neu-ropathy (DAN) is a major contributing factor in the morbidity of silent myocardial ischemia and infarction among patients suffering from DM.
\end{abstract}

Keywords: Diabetes Mellitus; Myocardial Infarction; Coronary Artery Disease; Diabetic Neuropathy; Diabetic Autonomic Neuropathy; Cardiac Autonomic Neuropathy.

\section{Introduction}

DM is a group of metabolic diseases characterized by hyperglycemia resulting from defects in insulin secretion, insulin action, or both (Association 2009, WHO $\mid$ About Diabetes 2014). The chronic hyperglycemia of diabetes is associated with long-term damage, dysfunction, and failure of different organs, especially the eyes, kidneys, nerves, heart, and blood vessels and if left untreated, diabetes can cause many complications. Acute complications can include diabetic ketoacidosis, hyperosmolar hyperglycemic state, or even death. Serious long-term complications may include cardiovascular disease, stroke, chronic renal disease, etc. Another long term complication of DM is the death or damage of nerve cells due to hyperglycemia, a condition called "Diabetic Neuropathy". DN is one of the most common complications of DM. The symptoms can include numbness, tingling, pain, and altered pain sensation. This condition, if left untreated plays a major role in the occurrence of atypical symptoms when a diabetic patient suffers an MI or myocardial ischemia (a condition known as silent MI) ( Hill 2014, G 2007, Chen et al.2016, Gandhi et al. 2017\& Feldman et al., 2019 ).

In 2014, DM affected about 3.5 million people in the United Kingdom alone, and about $50 \%$ to $60 \%$ of these patients suffered from DN. As of 2017, an estimated 425 million people had diabetes worldwide, with type-2 diabetes mellitus (T2DM) making up about $90 \%$ of the cases. This represents $8.8 \%$ of the adult population, with equal rates in both women and men( Drug Treatment of Diabetes Mellitus Endocrine and Metabolic Disorders, n.d.,Vos et al.2012). DN is a time-dependent condition, i.e. the longer the patient lives with the untreated diabetes, the more susceptible they become to developing the condition due to metabolic microvascular hypoxia caused by the patient's hyperglycemia.

Myocardial infarction (MI) is a major cause of death and disability worldwide. MI can be recognized by clinical features, including electrocardiographic (ECG) findings, elevated values of biochemical markers (biomarkers) of myocardial necrosis, and through imaging. MI is defined in pathology as myocardial cell death due to prolonged ischemia to the myocardium and it is a major cause of death and disability worldwide(Thygesen et al.2018). MI may be the first manifestation of coronary artery disease (CAD) or it may occur, repeatedly, in patients with established disease. In patients with known CAD and DM, the rate of death is greater than $70 \%$ over a period of 10 years. After MI has occurred, the 30-day mortality rate increases in patients with DM by more than $50 \%$, and of those who survive, approximately $50 \%$ die within five years after an MI, double the rate found in non-diabetic patients (Haffner et al.1998, Herlitz et al. 1998 \& Miettinen et al.1998).

The most common symptom is chest pain or discomfort which may travel into the shoulder, arm, back, neck or jaw. The discomfort may occasionally feel like heartburn and about $30 \%$ of people have atypical symptoms with women more often presenting without chest pain and instead having neck pain, arm pain or feeling tired. Among those over 75 years old, about $5 \%$ have had an MI with little or no history of symptoms (Task Force on the management of ST-segment elevation acute myocardial infarction of the European Society of Cardiology (ESC) et al.2012) Myocardial injury can also be detected when blood levels of sensitive and specific biomarkers such as cTn or the MB fraction of creatine kinase (CKMB) are increased. Cardiac troponin I and T are components of the contractile apparatus of myocardial cells 
and are expressed almost exclusively in the heart(Thygesen Kristian et al.2007). Most patients with MI present with severe clinical symptoms such as angina pectoris, but if the patient has no symptoms or atypical symptoms, the MI may be categorized as 'silent'(Dörr 2010). Silent MI is mostly very common among patients suffering from DM because of a condition known as DN, which comes about as a result of severe and prolonged diabetes. There are several medical articles and reviews that have explored the link between silent MI and DM, but the exact association between the two is not very well known. In this article review we intend to discuss the link between DM and its resulting DN, with the occurrence and frequency of silent MI and Ischemia, through the review of several published studies and articles.

\section{Pathogenesis of diabetic autonomic neuropathy}

The pathogenesis of DAN has not yet been clearly identified, but there are several published studies that support the concept that the pathogenesis of DAN differs between T1DM and T2DM. The main contributing factors that lead to the unset of DAN in T2DM include oxidative stress, vascular, and metabolic factors, whilst hyperglycemia is the main driving factor that leads to several destructive pathways in T1DM. Although there is currently no consensus on the matter, there are presently four main mechanisms thought to be the established causes of DAN.

Firstly, through increased Polyol Pathway Flux that affects several types of tissues in many sites in the body. By means of this pathway, increased activity of aldose reductase enzyme decreases the amount of Nicotinamide Adenine Dinucleotide Phosphate (NADPH). Increased flux through the polyol pathway may activate the Protein Kinase-C (PKC) pathway, and excessive activation of PKC leads to tissue damage. When sorbitol levels were increased in the blood and nerves of rat in certain experimental studies, it lead to harmful effects.

Secondly, hyperglycemia also results in increased formation of Advanced Glycated End-products (AGEs) through non-enzymatic glycation of mitochondrial respiratory chain proteins. This leads to the covalent bonding with proteins or lipids leading to intra and extracellular cross linking and aggregation thus causing deleterious effect on the nerves.

Third, through activation of PKC pathway. Protein kinase is a family of enzymes that control the function of other protein. They have the ability to affect collagen and growth factors, for example, they can transform growth factor B (TGF-B), leading to vascular occlusion followed by neuronal death. PKC is therefore a common mechanism for neuropathy and vascular disease.

Finally, through an increased Hexosamine Pathway Flux, which leads to the accumulation of the extracellular matrix as a result of hyperglycemia, which may in turn result in neuroinflammation associated with DAN( Niakan et al.1986, Schmidt 2002, Amara et al.2019\& Kallinikou et al. 2019) .

\section{Autonomic neuropathy and silent MI}

Clinical studies have confirmed an association between silent MI and silent ischemia, and autonomic neuropathy. Autonomic neuropathy is a group of symptoms that occur when there is damage to the nerves that manage involuntary body functions. These functions include blood pressure, heart rate, sweating, bowel and bladder emptying, and digestion. Autonomic neuropathy is a group of symptoms and it is not a specific disease. There are many causes of this condition with one of them being DN(Autonomic Neuropathy, n.d., Niakan et al.1986, Langer et al. 1991\&Wackers et al.2004 .

$\mathrm{DN}$ is nerve damage that is caused by diabetes. It is caused mainly by the increase of blood sugar levels over a long period of time, and also the high levels of fats, such as triglycerides, in the blood from DM, which damages your nerves. Symptoms may vary depending on the type of neuropathy. Peripheral neuropathy is a type of nerve damage that typically affects the feet and legs and sometimes affects the hands and arms. This type of neuropathy is very common. This condition can affect as many as 50\% of people with DM (Diabetic Neuropathy - Symptoms and Causes, n.d.). Autonomic neuropathy on the other hand is damage to nerves that control your internal organs, leading to problems with your heart rate and blood pressure, digestive system, bladder, sex organs, sweat glands, and eyes. The damage can also lead to hypoglycemia unawareness (Diabetic Neuropathy | NIDDK, n.d.). Major clinical manifestations of diabetic autonomic neuropathy (DAN) include resting tachycardia, exercise intolerance, orthostatic hypotension, constipation, gastroparesis, erectile dysfunction, sudomotor dysfunction, impaired neurovascular function, "brittle diabetes," and hypoglycemic autonomic failure.

The metabolic disorders of DM lead to diffuse and widespread damage of peripheral nerves and small vessels. Clinical manifestations of the autonomic dysfunction of DM and other microvascular complications frequently occur concurrently but in inconsistent patterns. The ubiquitous distribution of the autonomic nervous system (ANS) renders virtually all organs susceptible to autonomic dysfunction. Therefore, a patient diagnosed with DM should be suspected of having at least subclinical disturbances of the autonomic nervous system. Silent myocardial ischemia is one of the most common overt signs and symptoms of autonomic diseases caused by DM(Freeman 2014).

In a comparative study about silent myocardial ischemia in patients with T2DM and its relation with autonomic dysfunction, done by $\mathrm{R}$ Sukhija et.al, they showed that coronary angiography done in their test patients with silent ischemia revealed higher prevalence of multivessel involvement and diffuse disease in diabetics as compared to controls. And that half the diabetics (50\%) and none of their control had autonomic dysfunction. Autonomic dysfunction was present in $85.7 \%$ of diabetics with silent ischemia compared to $18.7 \%$ diabetics without silent ischemia, thus showing a link that autonomic dysfunction caused by DM is a major risk factor for the occurrence of silent myocardial ischemia, and ultimately silent MI(Sukhija et al.2000).

\subsection{Cardiac autonomic neuropathy (CAN) and silent MI}

Cardiovascular autonomic neuropathy (CAN) is a severely debilitating yet under-diagnosed condition in patients with DM. The prevalence can range from $2.5 \%$ (based on the primary prevention cohort in the Diabetes Control and Complications Trial) to as high as $90 \%$ of patients with type 1 diabetes mellitus (T1DM). Clinical manifestations range from orthostasis to MI. The diagnosis is made using multiple autonomic function tests to assess both sympathetic and parasympathetic function. The pathophysiology of CAN is complex, likely multifactorial, and not completely understood. Treatment is limited to symptomatic control of orthostatic hypotension, which is a late complication, and current strategies to reverse CAN are limited(Agashe \& Petak 2018)

CAN is perhaps one of the most overlooked of all serious complications of DM. It results from damage to the autonomic nerve fibers that innervate the heart and blood vessels and results in abnormalities in heart rate control and vascular dynamics. In a review of several epidemiological studies among individuals diagnosed with DM, it was shown that the 5 -year mortality rate from this serious complication is five times higher for individuals with CAN than for individuals without cardiovascular autonomic involvement(Ziegler 1994).

The development of CAN is associated with the lesion of the autonomic nervous system (ANS), and may be accompanied by coronary vessels ischemia, arrhythmias, "silent" MI, severe orthostatic hypotension $(\mathrm{OH})$ and sudden death syndrome. At the early stages CAN can 
be subclinical and it becomes clinically evident as the disease progresses. Based on the CAN Subcommittee of the Toronto Consensus Panel on Diabetic Neuropathy and the American Diabetes Association (ADA), CAN is defined as the impairment of cardiovascular autonomic control in patients with DM following the exclusion of other causes.

The main predictors for the development of CAN in patients with T2DM are age, gender, ethnicity and presence of microvascular complications [nephropathy, retinopathy, and peripheral neuropathy (PNP)](Serhiyenko \& Serhiyenko 2018).

The cause of silent myocardial ischemia in diabetic patients is controversial. It is clear, however, that a reduced appreciation for ischemic pain can impair timely recognition of myocardial ischemia or infarction and thereby delay appropriate therapy. There have been several studies undertaken that show the relationship between autonomic dysfunction and silent myocardial ischemia in diabetic individuals. In a published study by GaminiAmbepityia et. al, investigating exertional myocardial ischemia in diabetes and the influence of autonomic function, anginal perceptual threshold was measured in 32 diabetic patients and 36 non-diabetic control patients, all of whom had typical exertional angina. Although ST depression occurred earlier in the diabetic than in the non-diabetic group, they found that the anginal perceptual threshold in the diabetic group was delayed by a mean of 86 seconds with $95 \%$ confidence intervals of 53 to 119 seconds. Autonomic function tests were abnormal in the diabetic group, and in both groups regression analyses (using a third order polynomial) showed marked prolongations of anginal perceptual threshold as the heart rate responses to the Valsalva maneuver decreased to below the normal range. These data suggest that prolongation of the anginal perceptual threshold may be caused by autonomic neuropathy involving the sensory innervation of the heart. The study concluded that in patients with DM, anginal perceptual threshold is prolonged in association with autonomic and sensory neuropathy. And based on their data collected, the data suggested that altered perception of myocardial ischemia results from damage to the sensory innervation of the heart, representing a feature of DN (Ambepityia et al.1990).

In another published study by B Marchant et. al, investigating the role of subclinical autonomic impairment in diabetic patients with silent ischemia, 22 diabetic and 30 non-diabetic patients with proved CAD and a history of angina and ischemia on treadmill stress testing underwent clinical tests of autonomic function and measurement of 24-h heart rate variability. The investigation found that out of 52 patients that manifested ischemia during treadmill testing, only 36 patients experienced angina (angina group), whereas 16 did not (silent ischemia group). Diabetic and non-diabetic patients were similar in age and extent of CAD. The clinical tests conducted showed reduced parasympathetic function in the diabetic patients (Valsalva ratio $1.38+/-0.07$ vs. $1.60+/-0.06 ; p=0.007$ ). Patients in the silent ischemia group were more often diabetic $(33 \%$ vs. $63 \%, \mathrm{p}=0.05)$ and had prolonged time to ischemia on treadmill testing $(200+/-20 \mathrm{vs.} 271+/-$ $20 \mathrm{~s}, \mathrm{p}=0.03$ ). In addition, autonomic function was impaired in the silent group (supine/standing heart rate ratio $1.15+/-0.02 \mathrm{vs} .1 .05+/-$ $0.02, \mathrm{p}=0.002$ ). Subgroup analysis showed that abnormalities of autonomic function were confined to the diabetic patients in the silent group. The research concluded that subclinical neuropathy is an important cause of silent ischemia in patients with DM (Marchant et al.1993).

H Hikita et.al also published a study investigating the differences between diabetic and non-diabetic patients with silent myocardial ischemia. They had a total of 110 patients (15 diabetic and 95 non-diabetic). All patients underwent treadmill exercise testing and 24-hour ambulatory electrocardiographic recording. Their study concluded that a DN that affects the autonomic pain fibers that innervate the heart may be involved in the mechanism of silent myocardial ischemia in diabetics.

All of the afore mentioned studies and research papers show to justify the link between CAN in patients with DM and the occurrence of silent or asymptomatic MI.

\subsection{Diabetic autonomic neuropathy (DAN) and silent myocardial ischemia}

$\mathrm{DN}$ is nerve damage that is caused by diabetes, caused over time, due to high blood glucose levels, and high levels of triglycerides, in the blood from diabetes(Airaksinen, 2001). The autonomic dysfunction caused by this condition leading up to the onset of silent myocardial ischemia has been thoroughly reported in several studies. In a study published by D.P. Murray et.al looking at autonomic dysfunction and silent myocardial ischemia on exercise testing in DM, they found that silent myocardial ischemia on exercise testing is common among patients with DM and is associated with severe autonomic dysfunction(Murray et al., 1990). Another study by J.J. O'Sullivan et.al conducted five standard autonomic function tests on 41 men with DM. The tests included postural change in blood pressure, postural change in heart rate, heart rate response to deep breathing, heart rate response to Valsalva's maneuver, and blood pressure response to sustained handgrip. All patients underwent 24 hour ambulatory electrocardiographic monitoring to detect silent ischemia. The tests concluded that autonomic neuropathy may prevent the development of anginal pain and thus obscure the presence of ischemic heart disease, with a relative risk of 42.2 (95\% CI 4.5 to 39.4, p less than 0.001) for the men with DM(O'Sullivan et al.1991).

\section{Diabetes mellitus (DM) and silent myocardial infarction}

To date, there have been several published studies conducted looking into the link between DM in connection to the occurrence of silent MI. During our research, we identified a few studies that show the link between patients that suffer from DM and the frequency of the occurrence of silent MI and we will highlight two of these studies here in this review.

In a study published in November 1986 called "Silent Myocardial Infarction And Diabetic Cardiovascular Autonomic Neuropathy", 73 consecutive diabetic adults with symptomatic peripheral neuropathy were evaluated for the presence of CAN and electrocardiographic evidence of MI. 25 (34.2\%) of the patients displayed symptoms of CAN, and $10(13.7 \%)$ patients had electrocardiographic evidence of MI. Of the 10 patients identified with MI, 7 were asymptomatic (silent) by history. The incidence of silent MI was significantly higher $(\mathrm{P}<.04)$ in patients with CAN. The study then postulated that sudden death in diabetic patients with CAN may be due to silent MI (Niakan et al.1986).

In July 1973, an 18 years follow up study titled "Clinical features of unrecognized myocardial infarction—Silent and symptomatic: 18 year follow-up: The Framingham study", was conducted in the city of Framingham, Massachusetts. In this study, the occurrence, prognosis and characteristics of persons with nonfatal unrecognized MI were studied prospectively in a population of 5,127 men and women and were followed up biennially for 18 years.

Of the 259 electrocardiographically documented MIs, 60 (23\%) were discovered only by routine ECG at the time of biennial examination. Of these unrecognized infarctions, $32(53 \%)$ were actually silent. Of the 32 patients judged to have a silent infarction, 22 reported interim illnesses that could have been compatible with MI. 10 of the 60 patients $(17 \%)$ reported no interim illnesses or symptoms. The Framingham study stated that patients with prior DM or high blood pressure appeared more likely to have unrecognized infarction, and also suggested that decreased sensitivity to pain may be an important factor in unrecognized MI. The study also pointed out that this decreased sensitivity to pain also increased the incidence of unrecognized MI in patients with DM (Bradley \& Schonfeld, 1962, Margolis et al.1973). 
It is important to note however that we found a paper published in 2001 by K. E. J. Airaksinen questioning whether asymptomatic CAD in diabetic patients is as a result of accelerated atherosclerosis rather than the more widely accepted autonomic neuropathy. He stated that although early clinical studies had suggested that diabetics with acute MI were more often present with atypical symptoms, later clinical studies have not confirmed this association. His claim was that the statistical power of epidemiological data is limited and does not provide proof of an association between DM and silent MI(Airaksinen 2001).

He also referenced studies done by L. Hume et.al and M. Juhani Koistinen et.al (Hume et al., 1986\&Koistinen et al., 1992), stating that they failed to show an association between abnormalities in conventional autonomic function tests and silent myocardial ischemia. Another study he referenced was one done by Eugene A. Caracciolo et.al which he claimed did not find any significant difference of asymptomatic ischemia during ETT and 48-hour AECG monitoring among patients with DM and the group without(Caracciolo et al.1996).

\section{Histological evidence}

In a study done by Isaac Faerman et.al investigating the histological evidence in the relationship between autonomic neuropathy and silent MI in diabetic patients, the authors studied the autonomic nerve fibers of the heart muscle with argentic and combined techniques, looking for lesions in the sympathetic or parasympathetic nerve fibers that conduct pain.

They concluded that the reason why diabetic patients suffer from silent MI could be due to a lesion of the afferent nerves that conducts the pain(Faerman et al.1977).

\section{Discussion}

A substantial number of MIs are asymptomatic or associated with minor and atypical symptoms, and are found accidentally during routine ECG examinations that reveal the existence of abnormal Q waves. Symptoms are often atypical or even absent, especially in diabetic patients. One of the most common reasons for this occurrence is the development of DN in patients with a long history of DM(Zellweger et al. 2004).

$\mathrm{DN}$ is the most common and troublesome complication of DM, leading to the greatest morbidity and mortality and resulting in a huge economic burden for diabetes care. It is the most common form of neuropathy in the developed countries of the world, and it accounts for more hospitalizations than all the other diabetic complications combined. It is also responsible for $50 \%$ to $75 \%$ of non-traumatic amputations. DN is a set of clinical syndromes that affect distinct regions of the nervous system, singly or combined. It may be silent and go undetected while exercising its ravages, which is what makes it a very dangerous condition. DN is therefore diagnosed by exclusion and even when DN is symptomatic, less than one-third of physicians recognize the cause or discuss this with their patients(Vinik et al.2013). Several studies have shown that in the presence of myocardial ischemia, diabetic patients report angina less frequently than non-diabetic patients, and also that the absence of angina is not equivalent to the absence of CAD in diabetic patients. Furthermore, atherosclerosis ( being the most common cause of myocardial ischemia and MI) accounts for $65-80 \%$ of all deaths among diabetic patients, compared with one-third of all deaths in the general population, with T2DM being associated with a substantially elevated risk of cardiovascular morbidity and mortality (Vinik et al. 2013).

The concept that silent MI is a characteristic of CAD in the presence of diabetes is originally based on the early report of Bradley and Schonfeld (Bradley \& Schonfeld, 1962). They found that diabetic patients who were admitted to hospital for MI more often had little or no pain during infarction than non-diabetic patients.

The asymptomatic forms of the myocardial ischemia and MI are also more common in diabetic subjects than in non-diabetic ones. Additional support for an association between autonomic neuropathy and silent myocardial ischemia has been provided by several studies that found diabetic patients experienced chest pain later after the onset of ST depression than non-diabetic patients and this delay was related to impairment in the tests of autonomic nervous function, as stated by a study conducted by K. E. J. Airaksinen et.al looking into silent CAD in patients with DM and asking whether it is a feature of autonomic neuropathy or accelerated atherosclerosis (Airaksinen 2001).

\section{Conclusion}

In conclusion, based on numerous published articles and studies conducted over the years, especially those highlighted on this article review, it is well established that autonomic neuropathy caused by prolonged DM is a major contributing factor to the morbidity of asymptomatic/ silent MI and silent myocardial ischemia in diabetic patients.

We therefore recommend that more research be done on CAN in diabetic patients in order to figure out its prevention, as it is already established that it is a major factor in the incidence of silent myocardial ischemia and infarction among diabetic patients.

\section{Conflict of interest}

The authors of this article review declare that there was no conflict of interest encountered in the writing of this review paper, and that no funding was received from any organization or company in regards to the writing of this article review.

\section{Abbreviations}

- Myocardial Infarction: MI.

- Coronary Artery Disease: CAD.

- Diabetes Mellitus: DM.

- Type-1 Diabetes Mellitus: T1DM.

- Type-2 Diabetes Mellitus: T2DM.

- Cardiovascular Disease: CVD.

- Diabetic Neuropathy: DN.

- Diabetic Autonomic Neuropathy: DAN. 
- Cardiac Autonomic Neuropathy: CAN.

\section{References}

[1] Agashe, S., \& Petak, S. (2018). Cardiac Autonomic Neuropathy in Diabetes Mellitus. Methodist DeBakey Cardiovascular Journal, 14(4), 251-256.

[2] Airaksinen, K. E. (2001). Silent coronary artery disease in diabetes-A feature of autonomic neuropathy or accelerated atherosclerosis? Diabetologia, 44(2), 259-266. https://doi.org/10.1007/s001250051609.

[3] Amara, F., Hafez, S., Orabi, A., El Etriby, A., Abdel Rahim, A. A., Zakaria, E., Koura, F., Talaat, F. M., Gawish, H., Attia, I., Abdel Aziz, M. F., El Hefnawy, M. H. M. F., Kamar, M., Halawa, M. R., El-Sayed, M. S., El Kafrawy, N. A., Khalil, S. H. A., \& Assaad, S. N. (2019). Review of Diabetic Polyneuropathy: Pathogenesis, Diagnosis and Management According to the Consensus of Egyptian Experts. Current Diabetes Reviews, 15(4), 340 345. https://doi.org/10.2174/1573399815666190226150402.

[4] Ambepityia, G., Kopelman, P. G., Ingram, D., Swash, M., Mills, P. G., \& Timmis, A. D. (1990). Exertional myocardial ischemia in diabetes: A quantitative analysis of anginal perceptual threshold and the influence of autonomic function. Journal of the American College of Cardiology, 15(1), 72-77. https://doi.org/10.1016/0735-1097(90)90178-R.

[5] Association, A. D. (2009). Diagnosis and Classification of Diabetes Mellitus. Diabetes Care, 32(Supplement 1), S62-S67. https://doi.org/10.2337/dc09-S062.

[6] Autonomic neuropathy: MedlinePlus Medical Encyclopedia. (n.d.). Retrieved 18 July 2020, from https://medlineplus.gov/ency/article/000776.htm.

[7] Bradley, R. F., \& Schonfeld, A. (1962). Diminished pain in diabetic patients with acute myocardial infarction. Geriatrics, 17, $322-326$.

[8] Caracciolo, E. A., Chaitman, B. R., Forman, S. A., Stone, P. H., Bourassa, M. G., Sopko, G., Geller, N. L., \& Conti, C. R. (1996). Diabetics with coronary disease have a prevalence of asymptomatic ischemia during exercise treadmill testing and ambulatory ischemia monitoring similar to that of nondiabetic patients. An ACIP database study. ACIP Investigators. Asymptomatic Cardiac Ischemia Pilot Investigators. Circulation, 93(12), 20972105. https://doi.org/10.1161/01.CIR.93.12.2097.

[9] Diabetic Neuropathy | NIDDK. (n.d.). National Institute of Diabetes and Digestive and Kidney Diseases. Retrieved 18 July 2020 , from https://www.niddk.nih.gov/health-information/diabetes/overview/preventing-problems/nerve-damage-diabetic-neuropathies.

[10] Diabetic neuropathy-Symptoms and causes. (n.d.). Mayo Clinic. Retrieved 18 July 2020, from https://www.mayoclinic.org/diseases-conditions/diabetic-neuropathy/symptoms-causes/syc-20371580.

[11] Dörr, M. (2010). Silent myocardial infarction: The risk beyond the first admission. Heart, 96(18), 1434-1435. https://doi.org/10.1136/hrt.2010.201384.

[12] Faerman, I., Faccio, E., Milei, J., Nuñez, R., Jadzinsky, M., Fox, D., \& Rapaport, M. (1977). Autonomic neuropathy and painless myocardial infarction in diabetic patients. Histologic evidence of their relationship. Diabetes, 26(12), 1147-1158. https://doi.org/10.2337/diab.26.12.1147.

[13] Freeman, R. (2014). Chapter 6-Diabetic autonomic neuropathy. In D. W. Zochodne \& R. A. Malik (Eds.), Handbook of Clinical Neurology (Vol. 126, pp. 63-79). Elsevier. https://doi.org/10.1016/B978-0-444-53480-4.00006-0.

[14] Gandhi, J., Dagur, G., Warren, K., Smith, N. L., \& Khan, S. A. (2017). Genitourinary Complications of Diabetes Mellitus: An Overview of Pathogenesis, Evaluation, and Management. Current Diabetes Reviews, 13(5), 498-518. https://doi.org/10.2174/1573399812666161019162747.

[15] Haffner, S. M., Lehto, S., Rönnemaa, T., Pyörälä, K., \& Laakso, M. (1998). Mortality from Coronary Heart Disease in Subjects with Type 2 Diabetes and in Nondiabetic Subjects with and without Prior Myocardial Infarction. New England Journal of Medicine, 339(4), 229-234. https://doi.org/10.1056/NEJM199807233390404.

[16] Hill, M. D. (2014). Stroke and diabetes mellitus. Handbook of Clinical Neurology, 126, 167-174. https://doi.org/10.1016/B978-0-444-534804.00012-6.

[17] Hume, L., Oakley, G. D., Boulton, A. J., Hardisty, C., \& Ward, J. D. (1986). Asymptomatic myocardial ischemia in diabetes and its relationship to diabetic neuropathy: An exercise electrocardiography study in middle-aged diabetic men. Diabetes Care, 9(4), 384-388. https://doi.org/10.2337/diacare.9.4.384.

[18] Kallinikou, D., Soldatou, A., Tsentidis, C., Louraki, M., Kanaka-Gantenbein, C., Kanavakis, E., \& Karavanaki, K. (2019). Diabetic neuropathy in children and adolescents with type 1 diabetes mellitus: Diagnosis, pathogenesis, and associated genetic markers. Diabetes/Metabolism Research and Reviews, 35(7), e3178. https://doi.org/10.1002/dmrr.3178.

[19] Koistinen, M. J., Airaksinen, K. E., Huikuri, H. V., Pirttiaho, H., Linnaluoto, M. K., Ikäheimo, M. J., \& Takkunen, J. T. (1992). Asymptomatic coronary artery disease in diabetes: Associated with autonomic neuropathy? Acta Diabetologica, 28(3-4), 199-202. https://doi.org/10.1007/BF00778998.

[20] Langer, A., Freeman, M. R., Josse, R. G., Steiner, G., \& Armstrong, P. W. (1991). Detection of silent myocardial ischemia in diabetes mellitus. The American Journal of Cardiology, 67(13), 1073-1078. https://doi.org/10.1016/0002-9149(91)90868-L.

[21] Marchant, B., Umachandran, V., Stevenson, R., Kopelman, P. G., \& Timmis, A. D. (1993). Silent myocardial ischemia: Role of subclinical neuropathy in patients with and without diabetes. Journal of the American College of Cardiology, 22(5), 1433-1437. https://doi.org/10.1016/07351097(93)90554-E.

[22] Margolis, J. R., Kannel, W. S., Feinleib, M., Dawber, T. R., \& McNamara, P. M. (1973). Clinical features of unrecognized myocardial infarction-silent and symptomatic. Eighteen year follow-up: The Framingham study. The American Journal of Cardiology, 32(1), 1-7. https://doi.org/10.1016/S0002-9149(73)80079-7.

[23] Miettinen, H., Lehto, S., Salomaa, V., Mähönen, M., Niemelä, M., Haffner, S. M., Pyörälä, K., Tuomilehto, J., \& Group, T. F. M. I. R. S. (1998). Impact of Diabetes on Mortality After the First Myocardial Infarction. Diabetes Care, 21(1), 69-75. https://doi.org/10.2337/diacare.21.1.69.

[24] Murray, D. P., O’Brien, T., Mulrooney, R., \& O’Sullivan, D. J. (1990). Autonomic dysfunction and silent myocardial ischaemia on exercise testing in diabetes mellitus. Diabetic Medicine: A Journal of the British Diabetic Association, 7(7), 580-584. https://doi.org/10.1111/j.14645491.1990.tb01452.x.

[25] Niakan, E., Harati, Y., Rolak, L. A., Comstock, J. P., \& Rokey, R. (1986). Silent myocardial infarction and diabetic cardiovascular autonomic neuropathy. Archives of Internal Medicine, 146(11), 2229-2230. https://doi.org/10.1001/archinte.1986.00360230169023.

[26] O’Sullivan, J. J., Conroy, R. M., MacDonald, K., McKenna, T. J., \& Maurer, B. J. (1991). Silent ischaemia in diabetic men with autonomic neuropathy. British Heart Journal, 66(4), 313-315. https://doi.org/10.1136/hrt.66.4.313.

[27] Serhiyenko, V. A., \& Serhiyenko, A. A. (2018). Cardiac autonomic neuropathy: Risk factors, diagnosis and treatment. World Journal of Diabetes, 9(1), 1-24. https://doi.org/10.4239/wjd.v9.i1.1.

[28] Sukhija, R., Dhanwal, D., Gambhir, D. S., \& Dewan, R. (2000). Silent myocardial ischaemia in patients with type II diabetes mellitus and its relation with autonomic dysfunction. Indian Heart Journal, 52(5), 540-546.

[29] Task Force on the management of ST-segment elevation acute myocardial infarction of the European Society of Cardiology (ESC), Steg, P. G., James, S. K., Atar, D., Badano, L. P., Blömstrom-Lundqvist, C., Borger, M. A., Di Mario, C., Dickstein, K., Ducrocq, G., Fernandez-Aviles, F., Gershlick, A. H., Giannuzzi, P., Halvorsen, S., Huber, K., Juni, P., Kastrati, A., Knuuti, J., Lenzen, M. J., ... Zahger, D. (2012). ESC Guidelines for the management of acute myocardial infarction in patients presenting with ST-segment elevation. European Heart Journal, 33(20), 2569-2619. https://doi.org/10.1093/eurheartj/ehs215.

[30] Thygesen, K., Alpert, J. S., Jaffe, A. S., Chaitman, B. R., Bax, J. J., Morrow, D. A., White, H. D., \& Infarction, T. E. G. on behalf of the J. E. S. of C. (ESC)/American C. of C. (ACC)/American H. A. (AHA)/World H. F. (WHF) T. F. for the U. D. of M. (2018). Fourth Universal Definition of Myocardial Infarction (2018). Journal of the American College of Cardiology, 72(18), 2231-2264. https://doi.org/10.1016/j.jacc.2018.08.1038. 
[31] Thygesen Kristian, Alpert Joseph S., White Harvey D., null null, null null, null null, null null, null null, null null, null null, null null, null null, null null, null null, \& null null. (2007). Universal Definition of Myocardial Infarction. Circulation, 116(22), 2634-2653. https://doi.org/10.1161/CIRCULATIONAHA.107.187397.

[32] Vinik, A. I., Nevoret, M.-L., Casellini, C., \& Parson, H. (2013). Diabetic neuropathy. Endocrinology and Metabolism Clinics of North America, 42(4), 747-787. https://doi.org/10.1016/j.ecl.2013.06.001.

[33] Vos, T., Flaxman, A. D., Naghavi, M., Lozano, R., Michaud, C., Ezzati, M., Shibuya, K., Salomon, J. A., Abdalla, S., Aboyans, V., Abraham, J., Ackerman, I., Aggarwal, R., Ahn, S. Y., Ali, M. K., AlMazroa, M. A., Alvarado, M., Anderson, H. R., Anderson, L. M., ... Murray, C. J. (2012). Years lived with disability (YLDs) for 1160 sequelae of 289 diseases and injuries 1990-2010: A systematic analysis for the Global Burden of Disease Study 2010. The Lancet, 380(9859), 2163-2196. https://doi.org/10.1016/S0140-6736(12)61729-2.

[34] Wackers, F. J. T., Young, L. H., Inzucchi, S. E., Chyun, D. A., Davey, J. A., Barrett, E. J., Taillefer, R., Wittlin, S. D., Heller, G. V., Filipchuk, N., Engel, S., Ratner, R. E., Iskandrian, A. E., \& Detection of Ischemia in Asymptomatic Diabetics Investigators. (2004). Detection of silent myocardial ischemia in asymptomatic diabetic subjects: The DIAD study. Diabetes Care, 27(8), 1954-1961. https://doi.org/10.2337/diacare.27.8.1954.

[35] WHO | About diabetes. (2014, March 31). https://web.archive.org/web/20140331094533/http://www.who.int/diabetes/action_online/basics/en/

[36] Zellweger, M. J., Hachamovitch, R., Kang, X., Hayes, S. W., Friedman, J. D., Germano, G., Pfisterer, M. E., \& Berman, D. S. (2004). Prognostic relevance of symptoms versus objective evidence of coronary artery disease in diabetic patients. European Heart Journal, 25(7), 543-550. https://doi.org/10.1016/j.ehj.2004.02.013.

[37] Ziegler, D. (1994). Diabetic cardiovascular autonomic neuropathy: Prognosis, diagnosis and treatment. Diabetes/Metabolism Reviews, 10(4), 339383. https://doi.org/10.1002/dmr.5610100403. 